\title{
Novel Method for Vector Mixer Characterization and Mixer Test System Vector Error Correction
}

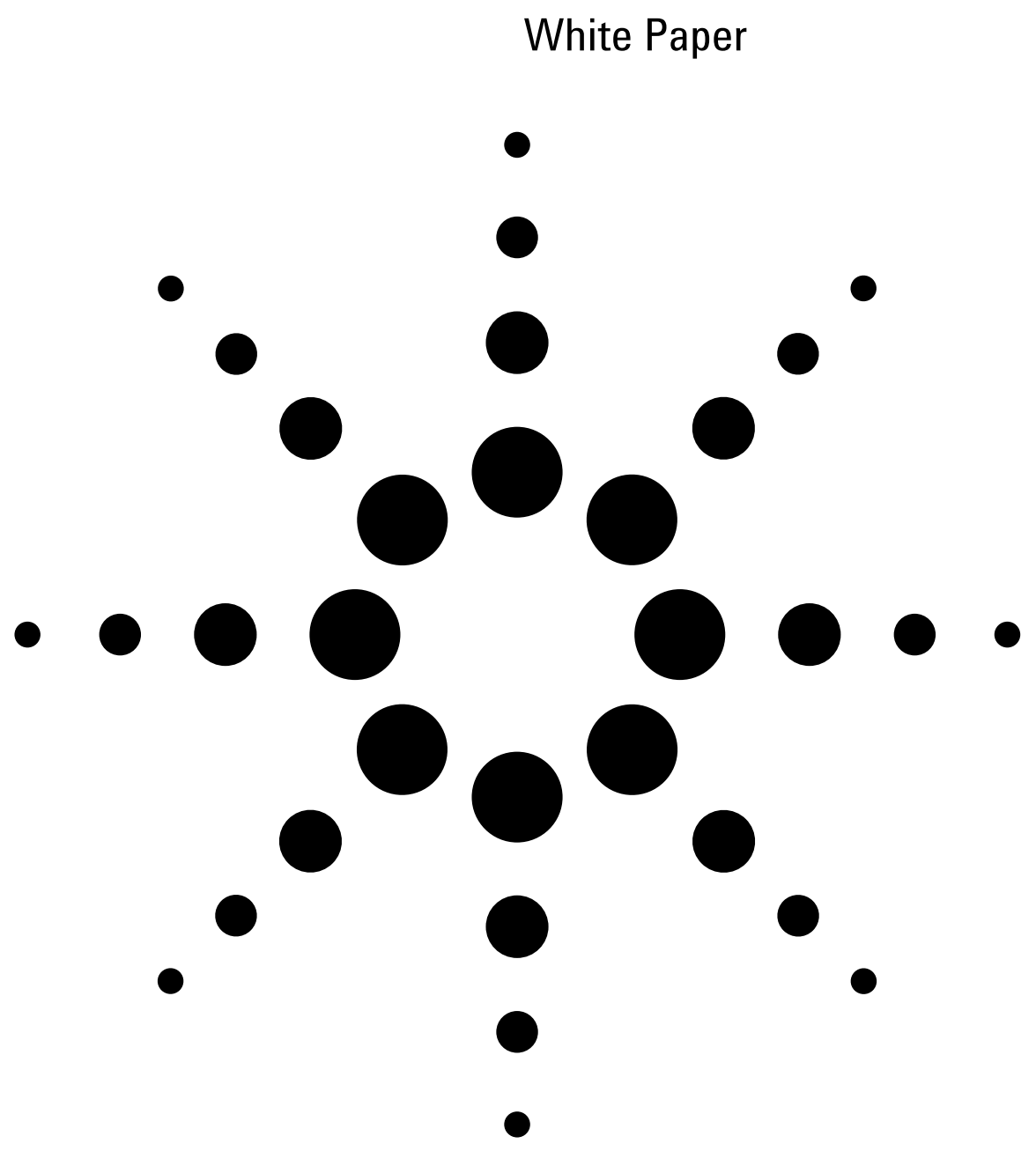


This paper presents a novel method for characterizing RF mixers, yielding magnitude and phase response for input match, output match, and conversion loss, and works for mixers which have reciprocal conversion loss and for which the image response can be filtered out. The characterized mixer is used to accomplish a full vector correction of a mixer test system, which can measure other mixers that are not reciprocal. A key contribution is phase and absolute group delay measurements of the mixer-under-test.

This paper was originally presented at the IEEE Microwave Theory and Techniques conference in June of 2002 [1].

\section{Introduction}

Many wireless and RF systems require frequency converters or mixers with specified and well controlled amplitude and group delay response. There are many techniques available to make amplitude response measurements, but phase or group delay measurements have proved very difficult.

A previous method described making group delay measurements by making three measurements on three pairs of mixers [2]. From these measurements, one can calculate the amplitude and phase response by solving the three linear equations for the overall response. This method makes use of up/down conversion but requires an IF filter between the pairs of mixers to avoid re-converting the unwanted side band. A second key aspect of this technique is that it assumes at least one of the mixers is reciprocal in its response, that is, it has the same conversion loss and group delay in the up-conversion mode as in the down-conversion mode. For some mixers, this holds approximately true.

A difficulty with this method is that it requires three sets of measurements, with reconnections between the mixer pairs and the filter. With each connection, there is room for random error (connector repeatability) and systematic error, for example, mis-match effects between the filter and mixer pairs, and between the mixers and test equipment. Mixers typically have poor return loss, so these mis-matches can be quite severe. It has been suggested that adding padding (attenuation) between the mixers and filters can reduce this effect, but it introduces further sources of mismatch between attenuators and the filter, and it reduces the levels of signal measured creating a noisy measurement. Finally, for frequency converters with internal Local Oscillators (LO), it may not be possible to drive the second of the pair of mixers with a phase locked replica of the LO, and thus it may not be possible to measure the output signal at the same frequency as the input signal.

An alternative method for characterizing the group delay (although not the phase or amplitude response) was presented by Knox [3]. This method relied on measuring the return loss of the mixer plus an airline, terminated in a short, over a broad range, and taking the time domain transform of the response. The time delay to the short response was subtracted from the length of the airline, to give the (two-way) delay of the mixer. This method had the difficulty of being appropriate only for broadband mixers, and the delay resolution was limited by the time domain resolution. Further, the delay response was a combination of response from both the sum and difference images. This method also assumed a reciprocal mixer. However, there was no need for additional mixers, or that the LO be locked to any of the signals. 


\section{Overview of the new method}

The novel method presented here can characterize the amplitude, phase, and delay response of a mixer, without the need to employ any other mixers. The input and output matches of the mixer are also determined.

This method does not require the LO signal of the test mixer to be accessible. The only requirement is a filter that can separate the response of the desired IF output signal from the unwanted side band. This method assumes a reciprocal mixer response, as do the previous methods, and any errors in this assumption will be errors in the final result. This method provides additional information on the input and output match of the mixer, which can be used in a later calibration step to remove instrumentation mismatch errors at the input and output of the test equipment. Because this does not require multiple mixer connections, connector repeatability does not add into errors of the measurement.

If a mixer can be characterized in terms of its input match, output match, and conversion loss (both magnitude and phase) then such a mixer can be used as a calibration device to calibrate a vector mixer measurement system [4]. Many such systems have been described, often including up/down conversion techniques, although calibrating the phase response of the system has always been an extremely difficult task [5].

Further, such measurement systems typically are not well matched, and no method has been demonstrated that corrects for the input and output mismatch effects of the calibration mixer, primarily because it was not possible to determine these characteristics. The vector calibration technique corrects for the mismatch effects of the input and output match of the calibration mixer, and can correct for the input mismatch of a mixer-under-test.

\section{Details of characterizing a calibration mixer}

The method requires a vector network analyzer (VNA). The analyzer is first calibrated for return loss measurement (S11) over the RF frequency range. This provides a "perfect" network analyzer (with only small residual errors). The mixer to be characterized is connected to this port, followed by the IF filter, as shown in Figure 1. Measurements of a metrology grade open/short/load are made (though any other one port calibration method will do) and these are saved. Note that the signals measured at the test port are a composite of the reflection from the mixer input port and a pair of converted signals: one un-desired, which is reflected of the IF filter and re-converted to the input signal; and another, which is the desired IF signal that passes through the IF filter and is reflected of the various standards used for a one-port calibration. 
The three measurements, $\Gamma_{O}, \Gamma_{S}, \Gamma_{L}$, are sufficient to calculate the "one-port" error model of the mixer/filter combination. That is, the values of $\Gamma_{O}, \Gamma_{S}, \Gamma_{L}$ can be used as inputs to the formulations for calculating the error terms of a one-port calibration.

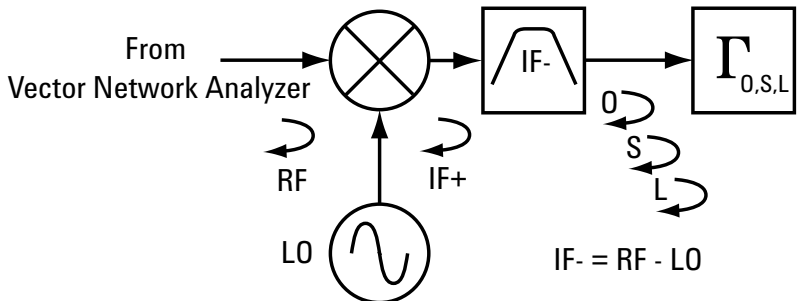

Figure 1: Mixer characterization diagram. Three measurements are made: open, short, and load response.

The one-port error model generates three terms:

1. Directivity: This term includes any reflections that do not change with the different applications of the standards. This term includes the reflection of the $\mathrm{RF}$ signal at the input to the mixer; and the signal that converts to the unwanted image, reflects off the filter, and reconverts to the RF signal. This unwanted image is not affected by the changing terminations, $\Gamma_{O}, \Gamma_{S}, \Gamma_{L}$ at the output of the filter. This is the input match of the characterized mixer, D.

2. Source Match: This term is the effective source match of the IF port including filter effects, M. This can be used in a later part of the through calibration to remove cal-mixer/port-match interactions at port 2 . This term essentially comes from the differences of the open reflection and the short reflection. Since only the desired IF image is affected by the changing termination, the effective source match at the output of the IF filter is determined.

3. Reflection Tracking: This represents the two-way insertion loss (magnitude and phase) through the mixer, $\mathrm{T} 1{ }^{*} \mathrm{~T} 2$. If the mixer is reciprocal in nature, taking the square root of this term gives the one-way insertion loss of the mixer, that is, $\mathrm{T} 1=\mathrm{T} 2$. The one-port error model already extracts the effects of the source match, and the calibrated VNA with which the data was taken includes effects of the input match. Thus, this term can be used as the actual two-way conversion loss of the mixer.

Since this data is taken with a calibrated network analyzer, the effective mismatch, tracking, and directivity of the VNA are eliminated. In practice, the one-port calibration function of the VNA can be used to extract the one-port error model of the mixer by downloading the previously measured $\Gamma_{O}, \Gamma_{S}, \Gamma_{L}$ responses into the VNA as the calibration standards of a one-port cal. However, remember that the standard data was taken over the RF range of the mixer, and while the standards are applied at the IF range, it is necessary to reset the analyzer frequency before the download to ensure the proper frequencies are used for the models of the calibration standard. Further, for cases where the RF is less than the LO frequency, it is necessary to reverse the data before downloading, so the response matches the "backward" sweep of the IF. However, the terms of the mixer can be found without using the VNA functions. Formulations for determining the extraction of Directivity, Source Match, and Tracking can be found in many references [6]. 


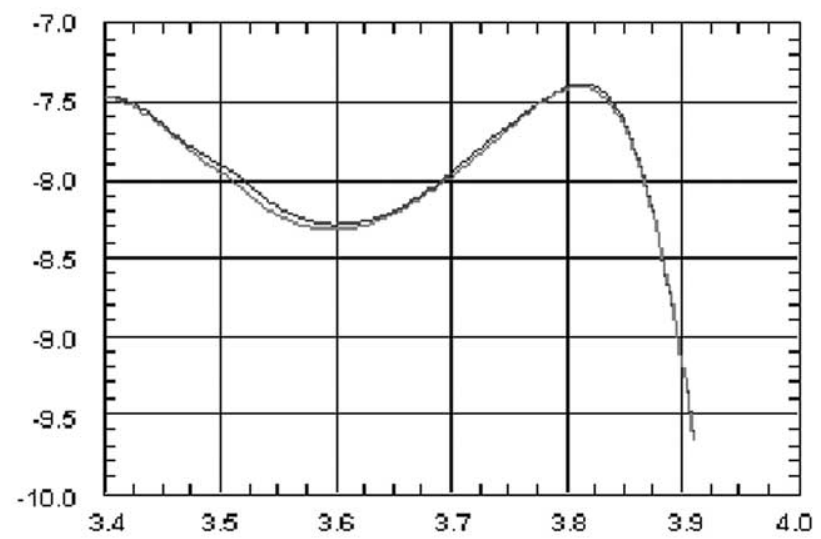

Figure 2a: Mixer conversion with the new method, measured as an up-converter and a down-converter.

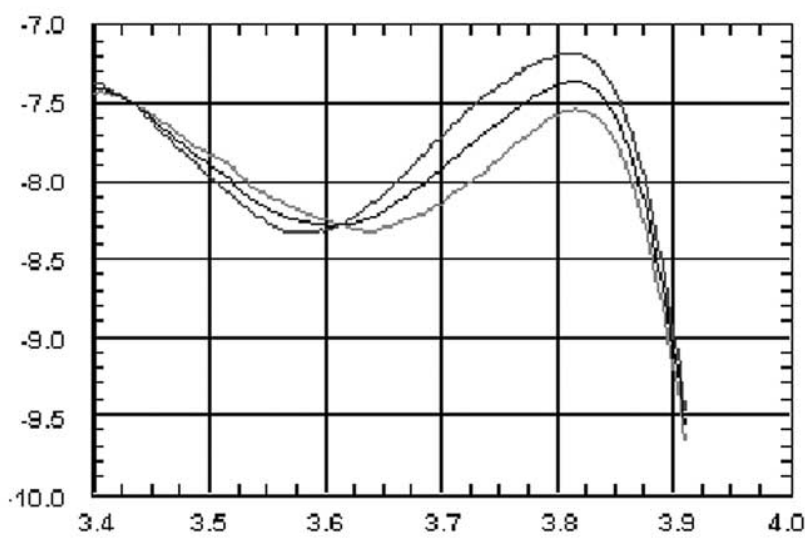

Figure 2b: Measuring the mixer with a power meter, with a third trace showing the average of up and down conversion.

Figure 2a shows the result of performing this characterization on a MiniCircuits ZFM mixer. A filter-mixer-filter combination was created, and characterized two times, once as an up-converter, and once as a down-converter. Figure $2 \mathrm{~b}$ shows the same measurements using a power meter calibrated VNA. In this measurement method, which can only measure the amplitude response, it is apparent that there is some non-reciprocal nature over a portion of the mixer's frequency response. However, the average of the up and down conversion is nearly identical to the response in Figure 2a, thus validating that the new method does indeed characterize the "round-trip" conversion loss of the mixer. If the amplitude non-reciprocity is treated as an error term, it would equate to approximately 1.2 degrees of phase non-reciprocity in this example.

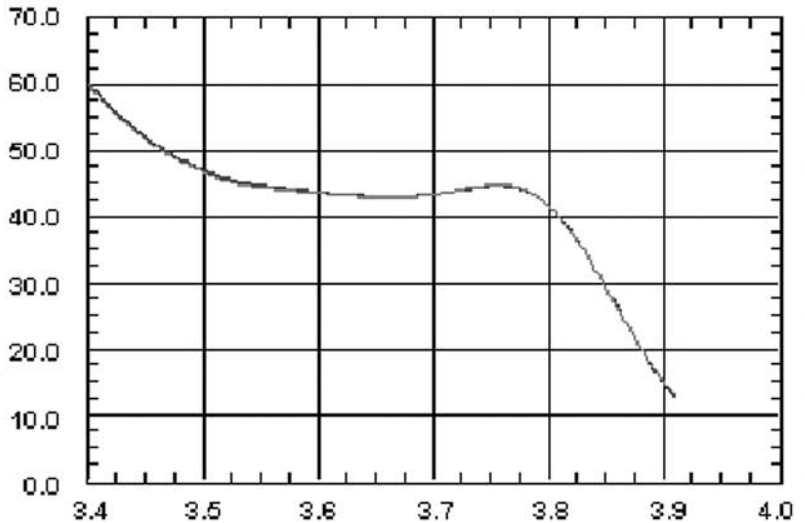

Figure 3a: Linear phase response of characterized mixer.

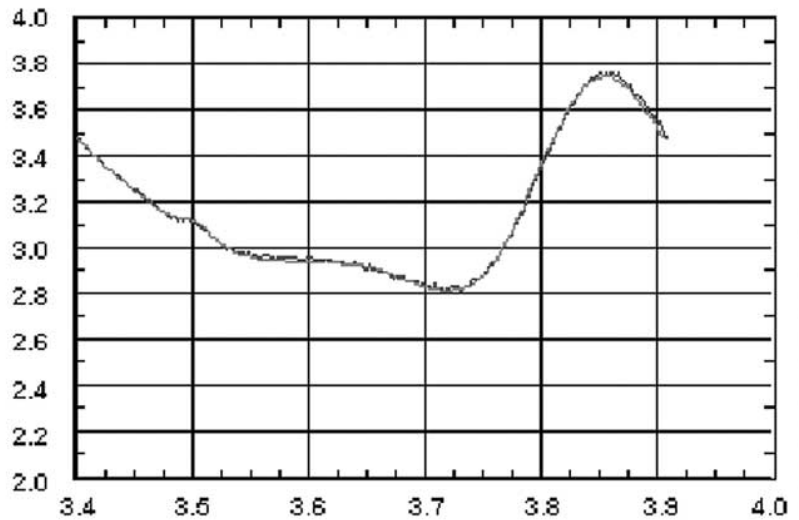

Figure 3b: Group delay response of characterized mixer; measured as both an up-converter and down-converter.

Figure 3 shows the phase and group delay measurements of the characterized mixer. (Phase measurements are normalized to remove the linear delay portion of the phase response). 


\section{Calibrating a test system}

The VNA can use the data of the characterized mixer as a starting point for calibration in a frequency-offset mode. The frequency-offset mode may use an additional mixer as either a down-converter (up/down mode) or as an $\mathrm{R}$-channel reference mixer (offset mode). To calibrate the VNA, a one port calibration over the IF range is used to measure the load match of port 2. This can be used with the source match data above to compensate for load match interaction. To properly compensate for the calibration mixer's match, the source match, ESF, and load match, ELF, of the system must be known. If the b2/a1 measurement of the calibration mixer is made (this is a raw, S21 measurement) then the transmission tracking error term, ETF can be computed by:

$E_{T F}=\left(S 21_{M 1} / T 1\right) \cdot\left(1-M \cdot E_{L F}-D \cdot E_{S F}-E_{S F} \cdot T 1 \cdot T 2 \cdot E_{L F}+E_{S F} \bullet M \bullet D \cdot E_{L F}\right)$

Where ELF and M are the load match and mixer output match at the output IF frequency, ESF and D are the VNA source match and mixer input match at the input RF frequency, T1 is the forward (up) conversion gain and T2 is the reverse (down) conversion gain. The VNA operating in offset mode measures the through response of the mixer, S21M1.

During measurement, the ELF, ESF, ETF are downloaded into the VNA error terms, and the standard two-port correction can be applied. If the reverse match of a DUT mixer is not measured, the ELF term can be set to zero, thus resulting in no compensation for the unknown mixer S22 element. 


\section{Evaluating the test system}

This test system error correction can be evaluated by measuring the same mixer, first by itself, and next with an airline (a device with low-loss, good match, but with delay). Ideally, the test system should show the conversion loss of the mixer reduced by exactly the loss of the airline. However, mismatch effects can cause extra ripple on the measurements. Figure 4 shows the result of measuring the mixer first without the airline, normalizing the trace, then again with the airline (darkest trace). Also shown is the same measurement but with a scalar calibration. The airline loss is nearly a flat line, as measured on a VNA in normal mode. Clearly, there is more than substantial improvement in the error of the measurement, as represented by the ripple.

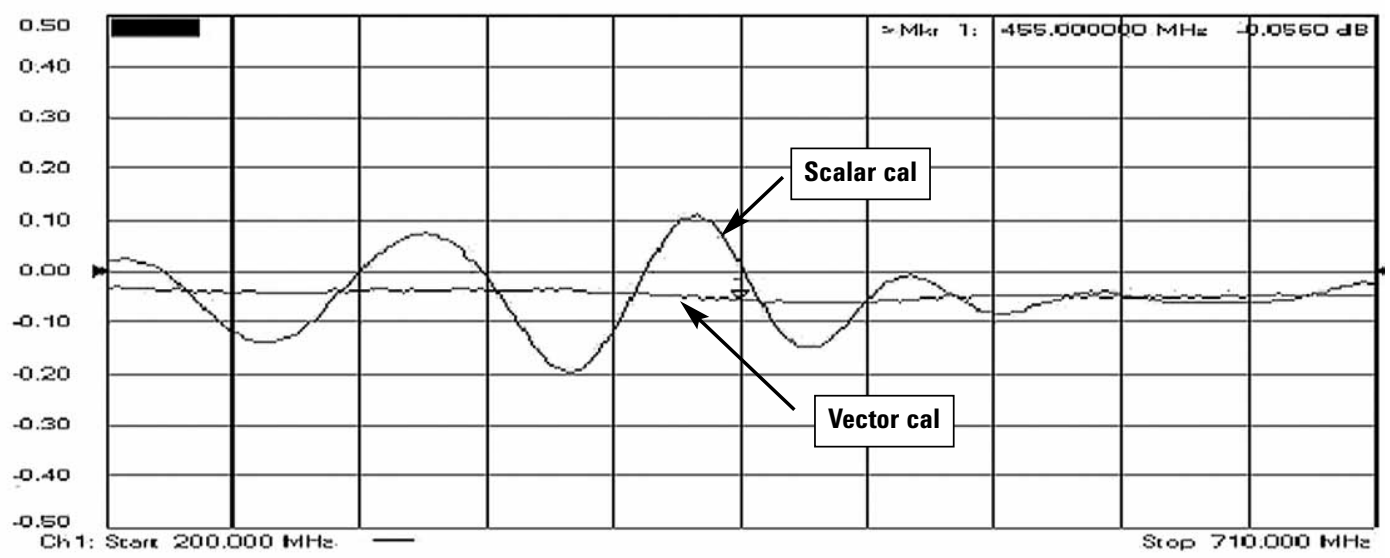

Figure 4: This shows the improvement in the mixer measurement of a mixer-plus-airline, as compared with a scalar calibration technique.

\section{Conclusion}

Calculating the group delay and phase response of a frequency-converting device such as a mixer has been a difficult challenge. The paper described a practical test system, which has high accuracy and fast measurement speed, with a reasonable calibration process. The first step of characterizing the mixer yields amplitude, phase and delay responses of the mixer with very simple measurements, and with no more restrictions than previously described techniques that are more complicated. The two-step process of first characterizing a calibration mixer, which must have reciprocal properties, then using that mixer to calibrate a more general frequency converting test system shows good results for example cases of mixer measurement. Further, a test system described provides for input and output vector correction of a mixer under test, which mixer need not be reciprocal. The uncertainty of the characterization of the calibration mixer is approximately the sum of the reflection tracking error of the calibration standards used for the VNA calibration, and the mixer characterization. 


\section{Acknowledgement}

The author wishes to acknowledge the assistance of Sean Hubert for the software development of the measurements of Figure 2a, and Dari Sariaslani in making the independent amplitude measurement shown in Figure $2 b$.

\section{References}

[1] Joel Dunsmore, Novel Method for Vector Mixer Characterization and Mixer Test System Vector Error Correction, 2002 IEEE_MTT-S International Microwave Symposium Digest, Vol 3, pp 1833-1836.

[2] C.J. Clark, A.A. Moultrop, M.S. Muha, C.P. Silva, Network Analyzer Measurement of Frequency-Translating Devices, Microwave Journal, Nov. 1996, pp 114-126.

[3] M. Knox, A Novel Technique for Characterizing the Absolute Group Delay and Delay Linearity of Frequency Translation Devices, Proceeds of the 53rd ARFTG Conference, June 1999.

[4] United States Patent 6,448,786, Stimulus/response System and Method of Vector Characterization of Frequency Translation Devices, September 10, 2002.

[5] Agilent 8753E Network Analyzer User's Guide, manual number 08753-90367, Chapter 3.

[6] Agilent 8753E Network Analyzer User's Guide, manual number 08753-90367, Chapter 6.

\section{Agilent Email Updates}

www.agilent.com/find/emailupdates

Get the latest information on the products and

applications you select.

For more information about Agilent Technologies test and measurement products, applications, services, and for a current sales office listing, visit:

www.agilent.com/find/tmdir

Product specifications and descriptions in this document subject to change without notice. Copyright (C) 2003 Agilent Technologies

Printed in U.S.A. November 11, 2003

5988-7826EN 